\title{
Aportes positivos de la informalidad laboral a la economía
}

pags 106-122

Grupo de investigación Desarrollo y Equidad

Línea de investigación: Economía social

Gustavo Adolfo Sandoval Betancour•

\section{RESUMEN}

Este trabajo busca identificar aportes positivos para las economías, asociados con las actividades de producción informal, analizando en particular el caso colombiano. Se encontró que, a partir de la gran crisis de fines de los años noventa en Colombia, la correlación entre formalidad e informalidad pasó a ser, de negativa a positiva, periodo a partir del cual se profundiza el análisis de la relación entre la producción formal e informal, permitiendo inferir un crecimiento simbiótico de los dos sectores. Además se concluye que el empleo informal aporta efectos positivos para la estabilidad y el crecimiento de la economía colombiana.

Palabras clave: informalidad laboral - segmentación mercado laboral - ingreso laboral.

\section{ABSTRACT}

This paper seeks to identify positive contributions to the economies associated with informal production activities, analyzing in particular the Colombian case. It was found that after the great crisis of the late nineties in Colombia, the correlation between formality and informality went from negative to positive. From that period the analysis of the relationship between formal and informal production deepens, inferring a symbiotic growth of the two sectors. Furthermore it is concluded that informal employment contributes positive effects for the stability and growth of the Colombian economy.

Key words: informal labor - labor market segmentation - labor income.

\section{INTRODUCCIÓN}

La informalidad laboral ha sido presentada reiterativamente como un gran problema social y económico, que se ha criticado e intentado combatir por diferentes razones.

Los puntos de vista de los autores que asumen esta postura son respetables y por lo general tienen razón en diversos aspectos. Pero también, en ocasiones son ligeros, o faltos de argumentos, o bien no se reconocen aspectos inherentes a dicho fenómeno que pueden considerarse positivos para la sociedad y la economía.

- Docente investigador de la Universidad de América. Mg.Ec. gustavo.sandoval@investigadores.uamerica.edu.co 
LÍNEA DE INVESTIGACIÓN: ECONOMÍA SOCIAL

La informalidad laboral, tanto en la época actual como en tiempos pasados, ha generado indudablemente, aportes positivos desde el punto de vista de la economía y el bienestar de la población. Precisamente, con este trabajo se busca hacer un análisis de algunos efectos positivos de índole económica, que se asocian a tal fenómeno, buscando que ello contribuya a moderar las posiciones respecto al tema y a discernir sobre lo que es positivo en él.

El desarrollo del trabajo parte de la identificación teórica, en la literatura sobre el tema, de algunas hipótesis respecto a los efectos económicos positivos de la actividad productiva informal. Dentro de éstas se destaca una hipótesis central, sobre la cual se avanzará para verificar su veracidad. Esta hipótesis propone que en virtud de conservar la estabilidad de las economías es preferible que exista la opción del trabajo informal en lugar de que se aumente el desempleo.

También se identificaron algunos casos contemporáneos de la economía empresarial en el caso colombiano, y de otros países, apropiados para ilustrar el esquema de acople de las actividades informales a la comunidad y a la economía formal. A partir de estos casos se hace un reconocimiento argumentado sobre los impactos positivos relevantes que se pueden atribuir a la actividad económica informal.

\section{LA INFORMALIDAD COMO FACTOR DE CAMBIO Y PROGRESO ECONÓMICO}

Las actividades informales de producción han constituido históricamente una fuerza decisiva de impulso hacia el cambio y el progreso. La incipiente transformación del desueto sistema feudal, imperante en Europa durante los siglos XI y XII, en una organización social más productiva y progresista, se debió al surgimiento del comercio entre las poblaciones europeas existentes entonces, actividad que nace en gran parte como consecuencia de las expediciones a Tierra Santa realizadas por los Cruzados.

El comercio, a la luz de las normas y costumbres existentes en esa época, se podría considerar una actividad informal, en cuanto no era habitual comerciar y tampoco usar el dinero para ello, dado que éste no hacía parte de las instituciones feudales. El comercio se extendió en lugares en que coincidían cruces de caminos o en desembocaduras de ríos que generaban tráfico de personas. Los comerciantes que se localizaron en estos sitios lo hicieron inicialmente de manera provisional en los burgos ${ }^{1}$ (Huberman, L.1996, pag.32), ya que no existían entonces sitios adecuados para tal fin. Con el tiempo la aglomeración de comerciantes hizo necesario expandir las áreas de localización a los fauburg (afuera del burgo) que con el tiempo se convirtieron en poblaciones.

A partir de esa época el uso del dinero se generalizó cada vez más, convirtiéndose en el medio de pago principal en las economías. Ello llevó también al surgimiento del crédito como fuente de financiación, aún en contravía de la doctrina de la iglesia católica.

A pesar de los duros choques ideológicos y de convivencia que se produjeron por las diferencias entre las costumbres e instituciones de propiedad y administración de los señores feudales con los comerciantes, a través del tiempo se acomodaron los unos a los otros mediante la inevitable asimilación de la actividad comercial, por el sistema hasta entonces vigente, con su consecuente transformación.

La narración de este pasaje histórico que hace Huberman, es un excelente ejemplo de cómo actividades consideradas como no tradicionales, o no institucionalizadas en el marco legal, de una u otra forma se abren paso cuando ellas hacen aportes positivos a la sociedad. El comercio como actividad informal en el momen-

1 Así se denominaban algunas zonas amuralladas de las ciudades. 
to de su aparición, debido a que no se contaba entonces con una reglamentación para su práctica, aportó a las economías de ese entonces y a las economías contemporáneas, una institución nueva que trajo condiciones muy importantes para lograr la superación de los niveles de desarrollo económico y de bienestar de esa época. En el mundo actual, el comercio y el dinero son parte imprescindible en cualquiera de los sistemas económicos existentes.

La lección que se puede sacar de lo anterior es: cuando las actividades productivas informales irrumpen en la economía para cambiar las instituciones, haciendo aportes significativos y mejorando el bienestar de la población, deben tomarse medidas para manejar la situación con acierto, porque lo más probable es que tales actividades llegan para quedarse. Eso implicaría hacer las reformas institucionales necesarias para acogerlas definitivamente.

Muchas de las actividades productivas contemporáneas calificadas de economía informal, han persistido durante años sin que muestren tendencias a reducir su magnitud, y por el contrario tienden a crecer en tamaño, lo cual ya es un indicador de que su aporte puede ser positivo y rentable socialmente. Son diversos en Colombia los casos de actividades informales que se comportan de esta manera y, sin embargo, en muchos casos no se hace un análisis técnico sobre la conveniencia de que ellas subsistan y se acojan en el largo plazo como parte del aparato productivo de la economía. Por el contrario es abundante la literatura que argumenta los aspectos negativos de la informalidad, en no pocos casos, sin fundamentos serios.

\section{REDUCCIÓN DE LA POBREZA POR LA INFORMALIDAD}

Es mejor que el desempleado trabaje en la informalidad a que no trabaje, porque puede obtener algunos ingresos que reducen su nivel de pobreza.
Esta frase resume los efectos que se pueden esperar de la informalidad laboral sobre la pobreza. No obstante, pareciera existir un entendimiento erróneo o por lo menos mal enfocado, pero muy difundido de lo que significa la informalidad laboral para una economía. Es común encontrar referencias que pretenden descalificar el aporte de la informalidad al bienestar, con base en argumentos simplistas, por ejemplo, destacando los bajos ingresos del trabajador informal. Al respecto Klein (1984, pag. 97) investigador del programa PREALC de la OIT señalaba:

"--- los estudios de los cuales se dispone sobre el tema sugieren una fuerte correlación entre la situación de pobreza y la pertenencia al sector informal urbano. En efecto, en Ciudad de México y en los principales centros urbanos de Colombia, el 73 por ciento de las personas que perciben ingresos inferiores al mínimo legal, estaban ocupadas en el sector informal; en Santo Domingo (República Dominicana) la proporción alcanza al 77 por ciento, en tanto que en San Salvador (El Salvador), en Asunción (Paraguay) y en centros urbanos del Perú el 80 por ciento o más de los urbanos pobres se encuentran en el sector informal".

Aunque es cierto lo que afirma el autor, se está desconociendo el aporte que hace la informalidad al bienestar de los desempleados, a pesar de que sigan siendo pobres.

También este tipo de miopía de apreciación se encuentra en estudios para el caso colombiano. En un ejemplo notorio, se puede consultarse a Galvis (2012, pag. 1), una publicación del Banco de la República (donde se afirma:

“--- una mayor incidencia de la informalidad se puede asociar con menos ingresos para los hogares, lo que afecta el bienestar general de la sociedad".

Y agrega el autor:

"La importancia del estudio de la informalidad está atada a que, para efectos de política 
LÍNEA DE INVESTIGACIÓN: ECONOMÍA SOCIAL

económica, ésta es perjudicial para un país en la medida en que altos grados de ésta pueden significar baja productividad, poca innovación, reducida recaudación de impuestos o evasión, así como un grado reducido de aportes a seguridad social" (ídem, pág. 5).

Esta afirmación es muy relativa, puesto que el grado de perjuicio social depende de las causas que presionan el crecimiento de la informalidad. En la literatura sobre informalidad, dependiendo de la fuente y de la definición que de ella se haga, se han identificado diversos tipos de informalidad laboral y de sus causas. A continuación se hace una breve reseña de cinco tipos de informalidad laboral con base en Castaño y García (2007):

\section{Informalidad subjetivista o voluntarista}

El concepto se atribuye a Maloney (2004) quien sostiene que la población que trabaja informalmente lo hace porque le conviene más hacerlo así, obteniendo mayores satisfacciones que si lo hiciera de manera formal. Propone pensar en aquellos trabajadores que presentan condiciones de competencias laborales muy bajas, quienes no tendrían posibilidades de un ingreso mayor en un trabajo formal que el que obtienen laborando informalmente. Por tanto, este tipo de informalidad no es por causas residuales de mano de obra, y tampoco significa que estos trabajadores vivan necesariamente peor que si trabajaran en un puesto formal.

\section{Informalidad paradójica}

Se reconoce a Portes y Haller (2004) como proponentes de esta causa de informalidad laboral, argumentando que, la cantidad de normas y leyes que regulan el trabajo formal, representa una invitación a transgredirlas, por la población trabajadora. De otra parte, también influye la voluntad y capacidad con que el gobierno haga cumplir la normatividad y las leyes. De aquí concluyen que el tamaño de la informalidad en la economía es directamente proporcional a la cantidad de normas que restringen el trabajo informal, e inversamente proporcional al esfuerzo que realicen los gobiernos para reprimirla.

\section{Informalidad ortodoxa}

La causa de esta informalidad está asociada con lo que De Soto et al. (1987) señalan como una inclinación muy arraigada en la población, como es la de querer trabajar con su propio negocio formal, pero dado que cumplir con la normatividad existente es engorroso y costoso, terminan por crear un negocio por fuera de las normas regulatorias. Este tipo de causa se considera de tipo residual, es decir, que se genera por no existir puestos de trabajo formal suficientes.

\section{Informalidad estructuralista.}

La causa de informalidad laboral desde esta perspectiva está asociada con la incapacidad de la economía formal para generar el número de empleos suficiente para absorber a los desempleados, constituyendo así la informalidad un refugio para los desempleados. (Cimoli, Primi y Pugno (2006, pag.91 y ss.)

\section{Informalidad institucional.}

Bajo este enfoque se destaca, como causa de la informalidad, a la precarización del empleo existente, debido al esquema de complementariedad que se ha venido configurando entre los actores del proceso de producción. Se ha producido a nivel nacional e internacional, una división más fragmentada del trabajo entre las etapas y actores de la producción de bienes y servicios, con nuevos esquemas que entran al proceso de subcontratación inter-empresarial, lo cual fomenta la entrada de empresas pequeñas e informales a la cadena productiva (Piore y Sabel, 1984).

Teniendo en cuenta la anterior relación de causas de informalidad, la validez de afirmaciones como la de Galvis deben ponerse en duda para países poco industrializados donde predomina la informalidad estructural (o excluyente). 
Su existencia se puede atribuir principalmente a la incapacidad de la economía para generar la suficiente demanda de trabajo, y así absorber la mano de obra disponible. Esquema bajo la cual las opciones para el desempleado son seguir desempleado o trabajar independientemente como informal. Sin embargo no se puede soslayar que en países poco industrializados la informalidad está conformada por la confluencia de más de una de las causas señaladas, cambiando continuamente las proporciones que aporta cada una.

No faltan opiniones que le atribuyen a la informalidad laboral muy graves consecuencias económicas. Novick (2008, pag.1) anota:

“--- la informalidad laboral se presenta como una de las problemáticas más graves que afectan al mercado de trabajo, y al mismo tiempo como uno de los factores determinantes de la pobreza y la exclusión social".

Por otra parte en artículo publicado en el periódico de temas económicos Portafolio (2013) se dice:

“---La informalidad laboral es, según las autoridades y expertos en el tema, el mayor problema que enfrenta el mercado de trabajo, pues su productividad es muy baja, y por lo tanto su remuneración también; además, la mayoría de trabajadores están afiliados a la salud subsidiada y un porcentaje muy pequeño cotiza a pensiones".

Al respecto se destaca la debilidad de los argumentos para calificar la informalidad como el peor problema del mercado de trabajo, frase que se ha convertido en un lugar común, que califica la informalidad laboral como un problema, inclusive peor que el mismo desempleo.

Respecto al flagelo que representa el desempleo para la fuerza de trabajo, en Colombia, los más golpeados por este son los más pobres, lo cual se refleja en la participación de los estratos, bajo y medio bajo, en el total de desempleados en el año 2000. Allí aportaban el $82 \%$, siendo las tazas de desempleo para los mismos estratos, en marzo de ese año, el 22,3\% y $20,9 \%$ respectivamente. En tanto que la tasa para los estratos, medio alto y alto, eran de $15,3 \%$ y $7,9 \%$ respectivamente (Sierra, 2001, pag.35).

En Portafolio (2009) se alerta sobre las consecuencias de la informalidad. Se señala allí que estudios de la OIT y la OMC analizan el impacto de la informalidad sobre las economías de los países en desarrollo, a la luz de la globalización. Entre sus efectos en las economías de países en desarrollo, se destacan

“---la baja diversificación de las exportaciones, la ampliación de la trampa de pobreza, la inflexibilidad para transitar de la economía informal a la formal y la ausencia de respuesta de la informalidad al crecimiento económico"

Además se sugiere que estas características están presentes en la economía colombiana. Evidentemente es poco probable que estos efectos dependan significativamente de la informalidad en las economías, pareciendo más bien que tienen que ver con problemas de eficiencia y productividad del empresariado formal, de las estrategias para impulsar las exportaciones y de las políticas que aplica el gobierno para inducir la formalización de la producción informal.

En contraste con la visión sobre la informalidad arriba comentada, hay estudios que muestran que ella puede rendir importantes beneficios a las economías si se sabe cómo aprovecharlos. Bigsten et al. (2004), refiriendose al caso africano en Kenia, comparan los beneficios de la informalidad con los costos y los riesgos de operar al margen de la legalidad Llegan a la conclusión de que, dado el entorno comercial existente, la permanencia en el sector informal puede ser una opción racional para los empresarios africanos, ya que reduce sus costos sin afectar la productividad, pudiendo participar más intensamente en el comercio internacional. Igualmente, Ishengoma y Kappel (2006) llegan a la misma conclusión utilizando los datos disponibles para comparar 
LÍNEA DE INVESTIGACIÓN: ECONOMÍA SOCIAL

los costos respectivos de la formalización y la informalidad.

\subsection{Dónde se localiza socialmente la informalidad en Colombia}

Cifras de la economía colombiana sobre los ingresos de los hogares permiten identificar su estructura por tipo de fuente y, espe- cíficamente, por concepto de ingreso. En la gráfica 1 se aprecia la importancia del ingreso mensual que se generó en un periodo de alto crecimiento de la economía (2006/2007), por los trabajadores independientes que se asimiIan a informales, clasificados por deciles de ingreso, para el total nacional de los hogares.

Gráfica 1. Distribución del ingreso corriente monetario de los hogares colombianos según deciles, por fuentes del ingreso - 2006/2007.

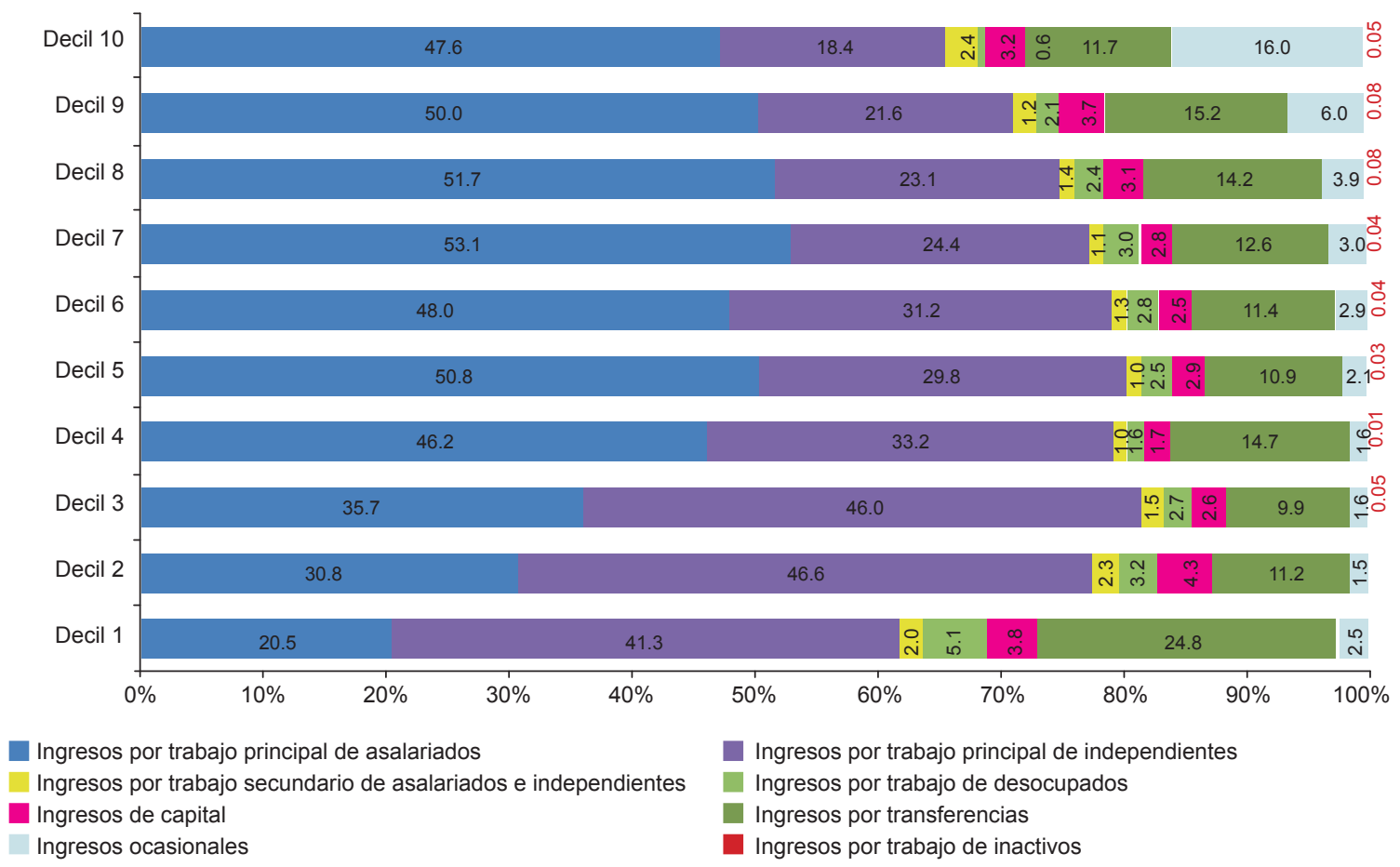

Fuente: DANE, ENIH 06/07

Se observa en primer lugar que en los tres primeros deciles (ingresos más bajos) de los hogares, la mayor fuente de ingresos, que representan más del $41 \%$ del total, corresponde a ingresos de los trabajadores independientes o informales. Aún en los niveles de mayores ingresos (deciles 8 a 10) la fuente de ingresos como trabajadores independientes genera una parte significativa de su ingreso total, siendo el $21 \%$ en promedio para los tres deciles de mayores ingresos. Para la totalidad de los hogares se generó en el mismo periodo, por trabajo independiente, el $23,3 \%$ del total de su ingreso monetario corriente.

La pregunta que se plantea ante las anteriores reflexiones es: ¿y si no fuese posible que los trabajadores desempleados pudieran obtener ingresos en empleos informales, estos no serían aún más pobres? La respuesta es obvia. Serían mucho más pobres, y el conjunto de pobres del país aumentaría. No sobra mencionar 
que la dependencia de los ingresos, provenientes del trabajo informal en los hogares de las zonas rurales, es mucho mayor, sobrepasando en promedio el $50 \%$ del ingreso en los cuatro deciles más pobres.

En la gráfica 2, se presentan las tendencias de mediano plazo de los ingresos totales de los hogares, en las trece áreas metropolitanas, y de los empleados formales e informales, en el periodo de recuperación económica 2001/06. En el periodo hubo crecimiento positivo de los ingresos en ambas categorías de empleo, aunque el crecimiento de los ingresos de empleados formales evolucionó más rápido que el de los informales. Lo que demuestran los datos es que estos últimos obtuvieron ingresos que les permiten sufragar gastos fundamentales para sus hogares. Cifras promedio de ingresos laborales per cápita indican que los empleados informales percibieron una proporción del ingreso de los empleados formales que fluctuó alrededor del $50 \%$ entre 2007 y 2013 (Boletín DANE, varios años).

\section{Gráfica 2. Colombia: Ingresos de empleados formales, informales y total- 13 áreas metropolitanas}

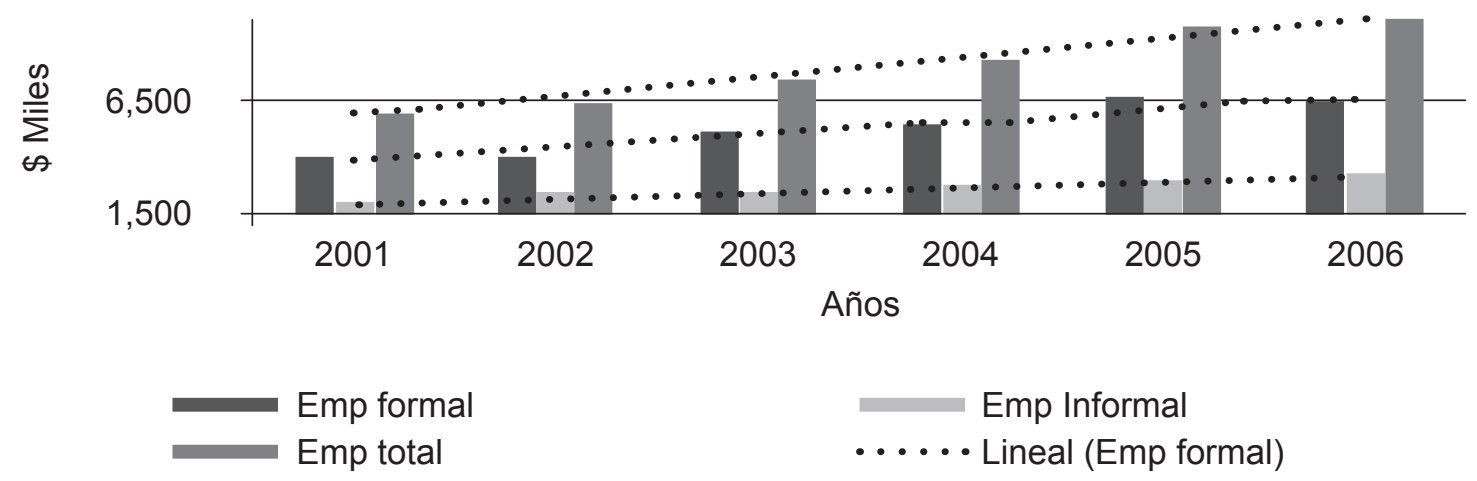

Fuente: Elaborado con base en boletines DANE - ECH de varios años.

Nota: Los ingresos totales incluyen otros conceptos diferentes a los laborales.

Otro aspecto que vale la pena señalar es la inelasticidad de la participación de los ingresos de fuentes de trabajo informal, frente a las fluctuaciones de la economía. Esto, sugiriendo que la informalidad laboral evita la profundización de la pobreza en los estratos más pobres, y entre los desempleados en épocas de desempleo creciente.

En la gráfica 3 se observa que en el periodo comprendido entre el primer semestre de 1982, y el primer semestre de 1992, el quintil de mayores ingresos disminuyó notoriamente su participación, periodo en que la tasa de crecimiento de la economía varió sustancialmente en cortos periodos.

Sin embargo la participación de los deciles de menores ingresos conservaron y aún aumentaron su participación en los ingresos laborales. Este comportamiento puede explicarse por el efecto de informalización de nuevos desempleados en periodos de bajo crecimiento de la economía, que induce el aumento del número de trabajadores informales $y$ el aumento del ingreso agregado de este segmento laboral.

No obstante, y a pesar de lo anteriormente observado, si bien la informalidad laboral alivia en alguna medida la situación de pobreza de los desempleados, se observa también que la distribución del ingreso empeora en el largo plazo pari passu, con el crecimiento de la economía (gráfica 4). Este comportamiento de la distribución y del crecimiento económico también es confirmado para Latinoamérica por Morely (2000, pág. 38). 
LÍNEA DE INVESTIGACIÓN: ECONOMÍA SOCIAL

Gráfica 3 - Distribución de ingresos laborales de perceptores por quintiles 1976 - 1996 (\% de participación en total ingresos)

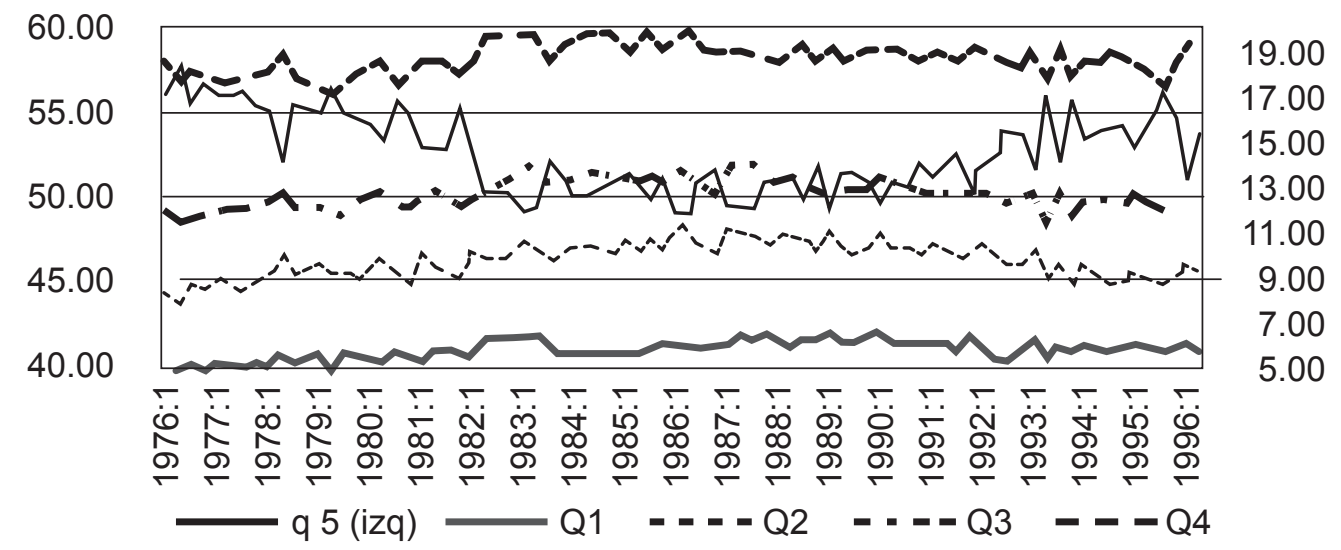

Fuente: elaborado con datos del DNP.

\section{Gráfica 4 - Coeficiente de Gini y PIB (Millones de pesos de 1994)}

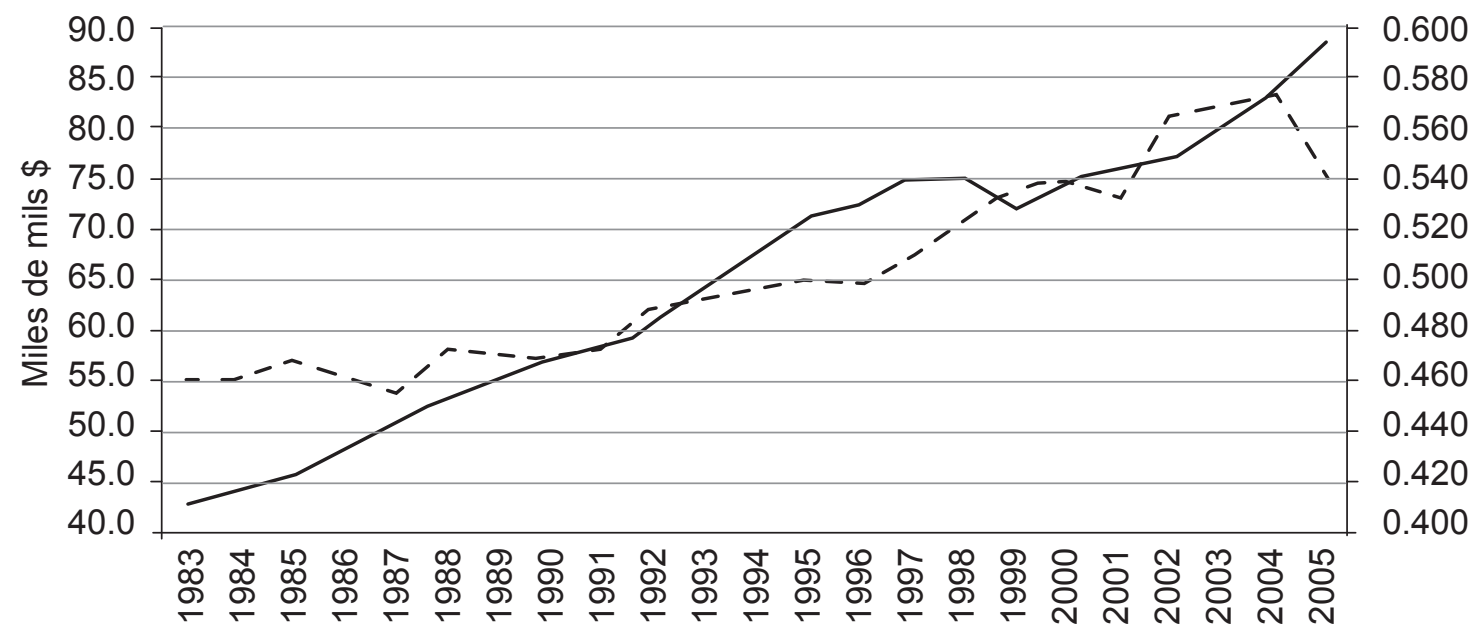

PIB - - - Coef Gini

Fuente: elaborado con datos del DNP.

El análisis de causalidad de granger, y de correlación entre el PIB y el coeficiente de Gini en el caso colombiano para el periodo 1983- 2005, indica que existe una correlación muy alta entre las dos variables, con un nivel de significación del 1\% (Véase anexo 1). Además, el análisis sugiere que el coeficiente Gini no es causante de la evolución del PIB, luego se acepta la hipótesis de que es más probable que el PIB sea causante de la evolución del coeficiente GINI con dos periodos de rezago (anexo 2).

\subsection{Efectos macroeconómicos de la informalidad laboral}

Las consecuencias de la economía informal van mucho más allá de la visión microeconómica, o lo que esto implica al nivel de personas o familias consideradas individualmente. El efec- 
to de la reducción de la pobreza se magnifica a través del impacto que tiene el trabajo informal a escala macroeconómica. A este nivel se pueden identificar consecuencias positivas que afectan directamente aspectos tan inesperados como el crecimiento de la economía, el éxito de las empresas formales, o el éxito o fracaso de un gobierno.

Desde el punto de vista macroeconómico, a continuación se aborda el análisis de los efectos de la informalidad sobre el desempleo. Aunque suene extraño, el empleo informal favorece el crecimiento del empleo formal como resultado de varias interrelaciones, por ejemplo, a través del efecto multiplicador del ingreso de los informales, y su consiguiente gasto en consumo; o a través de la reducción de costos de la industria formal por el encadenamiento que se ha generado con la producción informal. Con este fin se ha utilizado como recurso metodológico la segmentación del mercado laboral en tres componentes: segmentos de la PEA (población económicamente activa) empleada formalmente, empleada informalmente y población desempleada.

\subsection{Marco teórico}

El marco teórico aquí utilizado incluye la segmentación del mercado laboral en oferta y demanda de empleos formales e informales ${ }^{2}$. Desde el punto de vista del ingreso se segmenta el ingreso y el gasto de empleados formales e informales; y el gasto en consumo de los desempleados.

El comportamiento del mercado laboral se analiza mediante la utilización de funciones lineales que representan cada una de las variables arriba mencionadas, como aparece en la gráfica 5.La construcción gráfica del modelo con base en Sandoval (1992) que no incluye

2 La oferta de empleos informales debe entenderse como la existencia de oportunidades que ofrecen las normas y leyes para que cualquier persona pueda iniciar una actividad productiva informal mientras no pise terrenos considerados delincuenciales. sector público ni sector externo, se inicia con el trazo de una función post-keynesiana de ingreso, y otra de consumo para empleados formales, denominadas $\mathrm{Yf}$ y Cf. A estas funciones agregamos las funciones de ingreso y consumo de los empleados informales respectivamente $\mathrm{Yi}$ y $\mathrm{Ci}$, obteniéndose la función agregada de ingreso de formales e informales $\mathrm{Yf}+\mathrm{i}$, y de consumo $\mathrm{C} f+\mathrm{i}$. También se traza una función de consumo para desempleados y PEI (población económicamente inactiva) denominada Cñ con origen en el vértice derecho y pendiente negativa. Esto, suponiendo que los desempleados y la PEI realizan gasto en consumo con ahorros y transferencias. Esta función Cñ la agregamos con Cf obteniéndose $\mathrm{C} f+\tilde{n}$, que es la función consumo conjunta para empleados formales y desempleados e inactivos, suponiendo que el ingreso de los productores por concepto de ganancias se asigna a la inversión. La intersección de esta función con la función de ingreso Yf nos indica un nivel de desempleo y de empleo, de equilibrio, señalado con $\tilde{N} 1$ y N1 respectivamente, donde el ingreso es igual al gasto en consumo en una situación en que el empleo informal se supone que es igual a cero.

Para evaluar el impacto del empleo informal sobre el nivel de ingreso y el empleodeterminamos la intersección de las funciones $\mathrm{Yf}+\mathrm{i}$ con $\mathrm{C} f+\tilde{n}+\mathrm{i}$ que nos señala los nuevos niveles de empleo y desempleo de equilibrio, en N2 y $\tilde{N} 2$ respectivamente, con existencia de empleo informal mayor que cero.

El análisis del comportamiento del modelo explicado indica claramente que la adición del ingreso y el gasto en consumo de los empleados informales incide en el crecimiento tanto del empleo total de N1 hasta N2, como en la reducción del desempleo desde Ñ1 hasta Ñ2. Podríamos denominar este efecto "la paradoja de la informalidad laboral y el desempleo" porque el aumento del empleo informal contribuye, a través del crecimiento del ingreso y de la demanda agregada, al crecimiento del empleo formal y a la reducción del desempleo. 
LÍNEA DE INVESTIGACIÓN: ECONOMÍA SOCIAL

Gráfica 5- Segmentación del mercado laboral y de la función consumo en empleo formal, informal y desempleo.

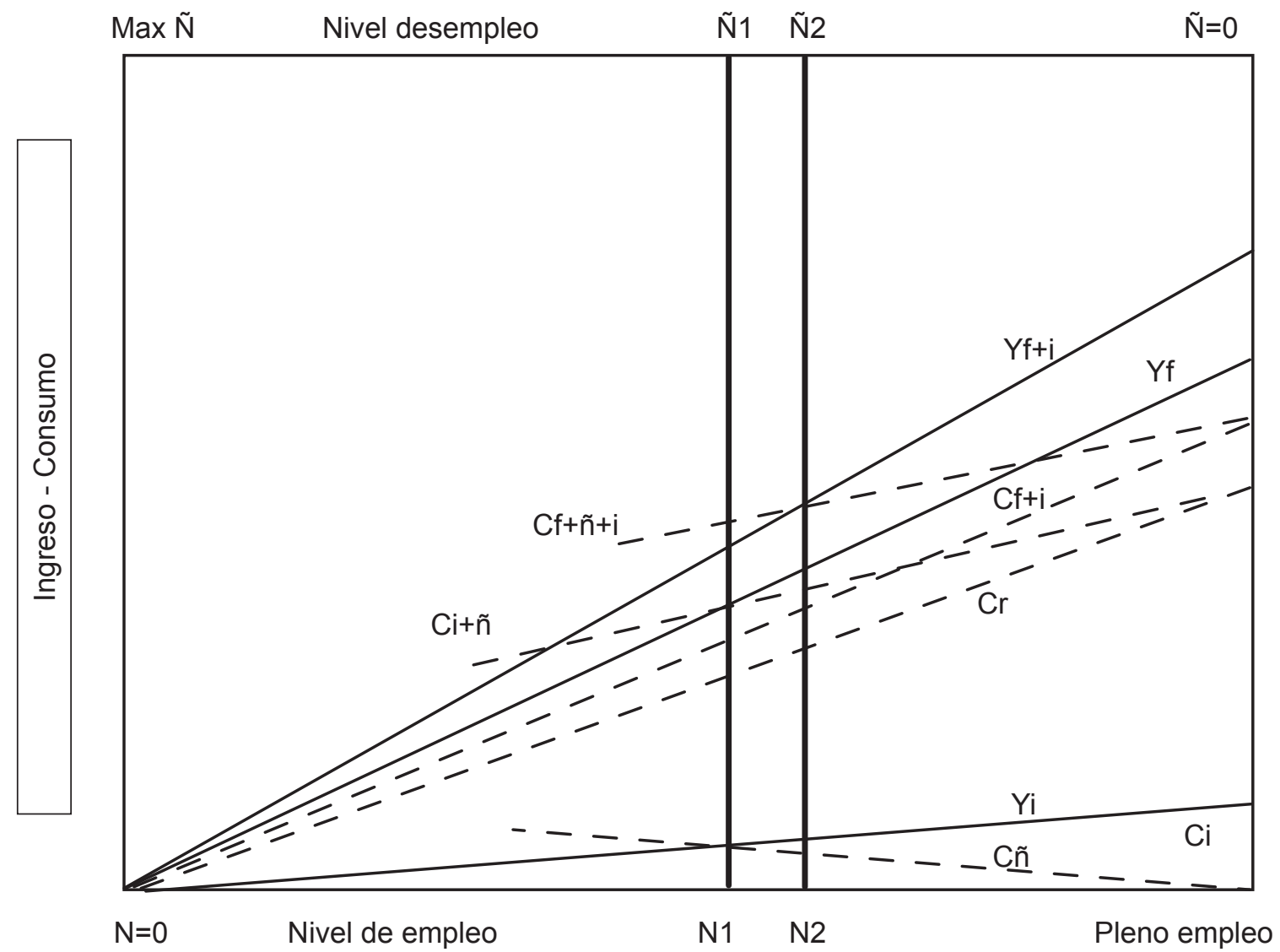

Notación: N Empleo - N Desempleo - I Informalidad - Cf Consumo de empleados formales - Ci Consumo de empleados informales - Cñ Consumo de desempleados - Yf Ingreso de empleados formales - YI Ingreso de empleados informales $\mathrm{Ni}$ Empleo de equilibrio - Ni Desempleo de equilibrio - Yf+i Ingreso de formales e informales - $\mathrm{C} f+i$ Consumo de formales e informales $-\mathrm{C} f+\tilde{n}$ Consumo de formales y desempleados $-C \mathfrak{f}+\tilde{n}+i$ Consumo de formales, desempleados e informales.

Fuente: El Autor.

Las reflexiones que se deducen del gráfico 5 sugieren que, teóricamente, debería presentarse cierto grado de correlación positiva entre el empleo formal y el empleo informal, a partir del supuesto de que la informalidad incide positivamente en el crecimiento del empleo formal.

Es importante mencionar que, además de los efectos positivos señalados mediante el modelo anterior, se debe tener en cuenta que la informalidad genera otros efectos económicos positivos a partir de su complementación con los procesos productivos formales

\subsection{La evidencia empírica}

Un argumento que destaca la importancia del ingreso y la demanda agregada, generados por el trabajo informal, se desprende de la composición del gasto en consumo total nacional de los hogares. El aporte que hace el trabajo informal e independiente al gasto de los hogares a nivel nacional osciló en el periodo 1994/05 alrededor del $40 \%{ }^{3}$. Ésta es una cifra muy significativa porque

3 Calculado con base en cifras del DANE- Gastos en consumo final- Cuentas de los hogares de las cuentas Nacionales. 
revela que, dada su participación, el trabajo informal tiene una gran capacidad para estabilizar o desestabilizar la economía colombiana. Ello corrobora a su vez que el trabajo informal contribuye de manera notable a la demanda agregada $y$, por lo tanto, es indudable que constituye un factor determinante para la creación o destrucción de empleo, formal e informal, a través de su respectivo efecto multiplicador.

Tokman (1978) analiza la relación comercial entre el sector formal e informal, y sugiere que hay efectos de estímulo al crecimiento de la producción formal a partir de la actividad informal. Harris (1990) también explora el tipo de relación entre la producción formal e informal y discute la autonomía del subsector informal, y su capacidad para crecer independientemente del subsector formal.

Con el caso de la India, (Janneke, P. et al., 2012) se ilustra más contundentemente la capacidad de la informalidad laboral para mantener la dinámica de las economías poco industrializadas. En el trabajo citado, a partir de un análisis econométrico, con datos de empresas formales, informales modernas, e informales tradicionales, se establece que el crecimiento del producto, en el sector industrial formal, está correlacionado significativa y positivamente con el crecimiento de la industria informal moderna. Sin embargo, no lo está con la informalidad tradicional, ni con el sector informal en conjunto. Esto indica que existe alta complementariedad entre la industria formal, y la industria informal moderna. También se encuentra que la creciente competencia, entre empresas formales, presiona la necesidad de reducir los costos, lo que se logra abasteciéndose de insumos o subcontratando producción con empresas informales del sector moderno.

Desde el punto de vista del ingreso laboral agregado, la participación de los trabajadores informales en el caso colombiano, representa también una parte muy importante del total. En el periodo 2001/06, su participación promedio fluctuó entre el $31 \%$ y el $36,7 \%$, en las 13 áreas metropolitanas (cuadro 1), lo cual corrobora lo ya ilustrado con su participación en el gasto.

Además debe considerarse que en las cifras anteriores, no se incluyen los ingresos de las zonas rurales, ni de las capitales departamentales más pequeñas, donde la participación del ingreso laboral informal representa una mayor proporción que en las áreas metropolitanas.

Vistos los ingresos laborales promedio por trabajador formal e informal, se encuentra que entre los años 2007 y 2013, la relación entre unos y otros es: Ios informales recibieron en el mismo periodo la mitad de lo recibido por los formales. (Véase cuadro 2)

Cuadro 1- Participación de Ingresos laborales de población ocupada formal e informal - 13 áreas metropolitanas (\%) 2001 - 2006 (abril - junio)

\begin{tabular}{|l|c|c|c|c|c|c|}
\hline \multicolumn{1}{|c|}{ Año } & 2001 & 2002 & 2003 & 2004 & 2005 & 2006 \\
\hline \% Ingreso formal & 67.3 & 63.3 & 67.4 & 67.1 & 69.0 & 65.5 \\
\hline \% Ingreso informal & 32.7 & 36.7 & 32.6 & 32.9 & 31.0 & 34.5 \\
\hline Ingreso total & 100 & 100 & 100 & 100 & 100 & 100 \\
\hline
\end{tabular}

Fuente: DANE - Gran Encuesta Integrada de Hogares (GEIH). 
LÍNEA DE INVESTIGACIÓN: ECONOMÍA SOCIAL

Cuadro 2. Promedio de ingresos laborales nominales de los ocupados formales e informales (En miles de pesos) - Total 13 áreas metropolitanas Trimestre abril-junio (2007 - 2013)

\begin{tabular}{|l|r|r|r|r|r|r|r|}
\hline \multicolumn{1}{|c|}{ Año } & $\mathbf{2 0 0 7}$ & $\mathbf{2 0 0 8}$ & $\mathbf{2 0 0 9}$ & $\mathbf{2 0 1 0}$ & $\mathbf{2 0 1 1}$ & $\mathbf{2 0 1 2}$ & $\mathbf{2 0 1 3}$ \\
\hline Empleo formal & 1129 & 1245 & 1124 & 1259 & 1296 & 1273 & 1409 \\
\hline Empleo informal & 589 & 565 & 587 & 635 & 621 & 633 & 642 \\
\hline \% (Infor/Form) & 52.2 & 45.4 & 52.2 & 50.4 & 47.9 & 49.7 & 45.6 \\
\hline
\end{tabular}

Fuente: GEIH. Tomado de Boletín DANE de informalidad laboral - abril junio 2013

En relación con el comportamiento del mercado laboral segmentado, en la gráfica 6 se presenta el empleo formal e informal y la tasa de desempleo durante el periodo 1992 a 2010. En los años noventa existió una diferencia notable en el comportamiento de correlación entre empleo formal e informal. Mientras que en los primeros 8 años de la década se movieron estas variables en sentido inverso, a partir del año 2000/01 empiezan a tener un comportamiento de largo plazo que indica crecimiento de las dos variables en el mismo sentido, lo que resulta compatible con la tesis de que el aumento del empleo informal contribuye a generar empleo formal. Aunque cabe también la hipótesis opuesta, es decir que el crecimiento del empleo formal favorece el crecimiento del empleo informal.
Los cambios sustanciales en el tipo de correlación entre las variables a partir de la crisis de los años 1998/99 sugieren que ésta afectó sustancialmente el comportamiento del mercado laboral. En efecto hasta 1999 la informalidad se movió con respecto a la tasa de desempleo y al empleo formal, de acuerdo con lo esperado: a bajas tasas de desempleo corresponde altos niveles de empleo formal y bajo nivel de empleo informal. Sin embargo a partir del fin de la crisis la tendencia creciente de largo plazo del empleo formal va acompañada de una tendencia en el mismo sentido del empleo informal. Específicamente en el periodo 2001/05, a pesar de reducirse el desempleo y aumentar el empleo formal, la informalidad mantuvo un crecimiento positivo, y lo mismo ocurre de 2008 en adelante.

\section{Grafica 6 - Empleo formal e informal y tasa de desempleo (\%)}

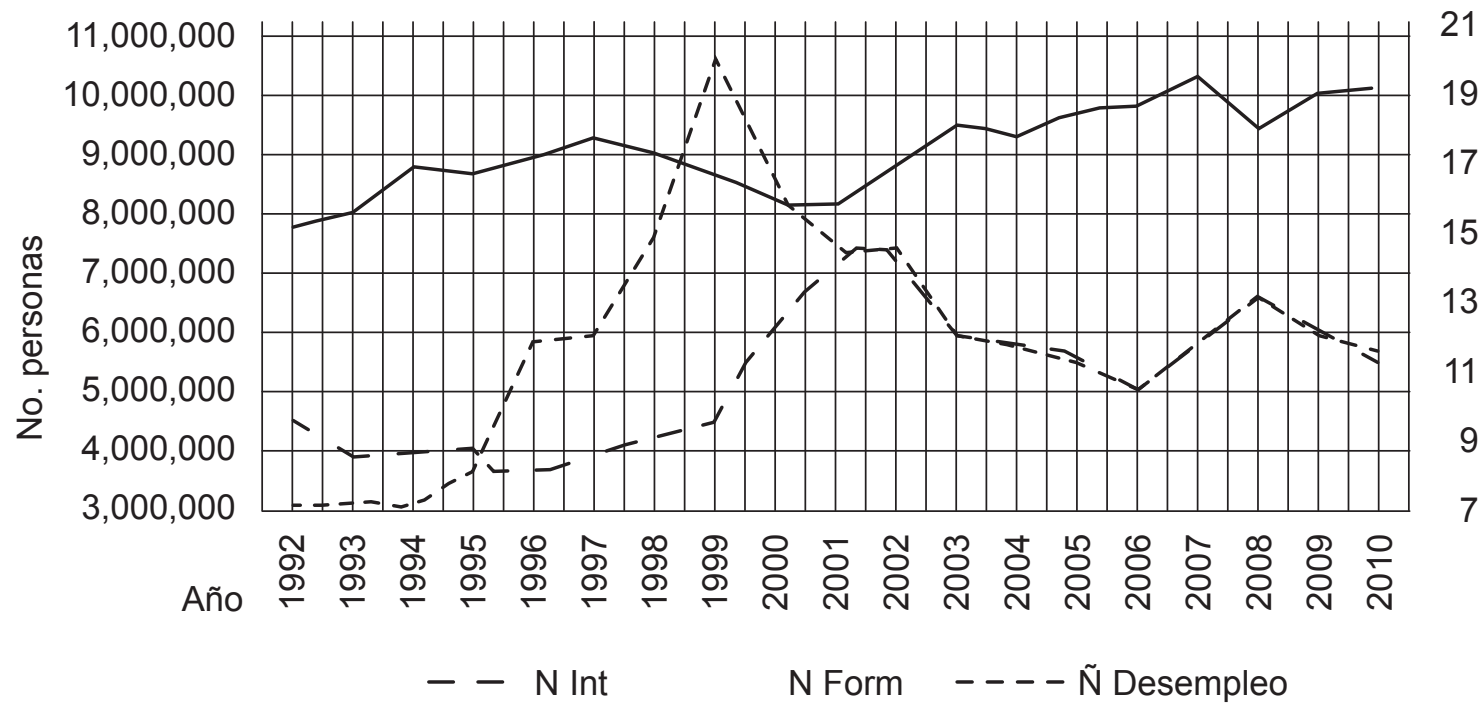

Fuente: DANE y página digital Banco Mundial / OIT 
De las observaciones anotadas sobre el comportamiento del mercado labora, se concluye. Primero, que la economía colombiana adoptó el trabajo informal como refugio para el desempleo, y como una institución sectorial más de la economía, complementaria con la economía formal, en dos sentidos: uno como como un proveedor de mano de obra que se requiera en el sector formal, en épocas de crecimiento económico o, dos, como lo ve la OIT (1972), fungiendo como un proveedor de insumos y bienes intermedios, cada vez más importante para la industria formal, y complemento del proceso productivo en la etapa de comercialización, surgiendo así una asociación simbiótica muy estrecha de los dos subsectores que obliga su crecimiento paralelo. Sin embargo la visión como reserva de mano de obra, parece no poseer capacidad para explicar el crecimiento simultáneo del empleo informal y formal. Así que la segunda visión de la relación complementaria, que parece haber conformado la producción informal con la economía formal, ofrece argumentos muy sólidos para sustentarse.

La tesis de la complementariedad productiva presagia su permanencia a pesar de los esfuerzos que se adelanten para su formalización (por ejemplo mediante lo previsto en la ley 1429 de 2010). Es por ello que la formalización de lo informal será siempre un esfuerzo que deberá hacerse, debido a que surgirán indefinidamente nuevas actividades de producción informal o, por lo menos, mientras exista desempleo involuntario. Así, la formalización por el Estado viene a reforzar uno de los principales aportes positivos de la informalidad como es la creación de nuevo empleo formal a partir del crecimiento del informal, lo que antes hemos Ilamado "la paradoja de la informalidad laboral y el desempleo".

Se debe puntualizar que la formalización por la acción del Estado es un refuerzo, porque por sí misma la producción informal da lugar a la generación de una serie de efectos económicos que impulsan el crecimiento de empleo formal. Es así, debido a que el trabajo informal de carácter estructural, aumenta la eficiencia conjunta de la PEA y, con ella, se aumenta el ingreso agregado y la producción de la economía, lo que a su vez da lugar a la ampliación de la demanda de trabajo formal e informal.

Es posible identificar casos de la economía colombiana en que los renglones manufactureros y de comercio prósperos, con una organización formal, realizan transacciones de compra de insumos y/o bienes intermedios provenientes de trabajo informal. Un ejemplo es el caso de empresas formales comercializadoras 0 procesadoras de chatarra que se abastecen de materiales provenientes de recicladores informales; empresas productoras de manufacturas de vidrio, cartón o plásticos, que reciclan insumos desechados como basura, recuperados con trabajo informal y reciclados por ellas. Esta clase de complementariedad entre lo formal y lo informal se fortalece cada vez más, impulsada en la medida en que se descubren nuevas técnicas de reciclaje y/o aplicaciones o usos para materiales reciclables. Otro tipo de complementariedad entre la economía formal e informal resulta del importante papel que desempeñan los trabajadores informales como canales de distribución y venta de la producción de bienes finales de industrias formales, y la lista de ejemplos de complementariedad de las cadenas productivas podría alargarse mucho más.

El tema lo discuten Ranis y Stewart (1999) llegando a concluir que efectivamente el potencial de crecimiento del subsector informal depende en gran parte del grado de complementariedad que tenga con el subsector formal. De otra parte Janneke, P. et al. (Op.cit.) analizaron el impacto que puede tener la subcontratación de producción del sector formal industrial con empresas informales en India, obteniendo resultados que apoyan la hipótesis de que los encadenamientos productivos, entre industria formal e informal, explican el crecimiento del empleo y el número de empresas informales del segmento más moderno de éstas. En consecuencia plantean la hipótesis de que el crecimiento correlacionado de la informalidad con la producción total de la economía también podría estar explicado por la modernización y mayor competitivi- 
LÍNEA DE INVESTIGACIÓN: ECONOMÍA SOCIAL

dad del subsector formal de la producción, que eleva la intensidad del capital creando mayores barreras al ingreso de nueva competencia, lo que llevaría al subsector informal más atrasado a especializarse en la producción de menor grado de complejidad. De otra parte se establece en el mismo estudio, que la subcontratación de la industria formal con la industria informal, ha tenido un gran efecto dinamizador de la economía de la India desde la liberalización de las políticas a comienzos de los años noventa. Como resultado de ello, actualmente la producción industrial informal ha llegado a representar el $90 \%$ del empleo en la industria manufacturera total (Janneke et al. pag. 21).

Se deduce de lo anterior que es razonable pensar que en la economía colombiana bien puede estar fortaleciéndose una complementariedad simbiótica de la producción manufacturera formal e informal, que estaría generando estímulos de crecimiento mutuo en los dos subsectores, explicándose por esta vía la correlación señalada entre el comportamiento del empleo en el subsector formal y el subsector informal.

\section{CONCLUSIONES}

La información analizada en el presente documento nos permite obtener algunas conclusiones referentes a la validez de ideas que han hecho carrera en la literatura sobre los efectos económicos de la informalidad laboral, y de su papel en las economías contemporáneas. Específicamente se espera haber demostrado con evidencia del caso colombiano que la informalidad laboral no necesariamente es una forma de producción perversa e incompatible con las políticas aplicables para elevar el nivel de bienestar en las sociedades modernas.

Una primera conclusión que resulta del trabajo es que la informalidad laboral contribuye, dada su importancia en el ingreso y el gasto, a la estabilidad de la economía nacional en Colombia.
También se concluye que la opción de trabajo informal tiende a reducir el impacto del desempleo sobre la población más pobre, a través del ingreso que perciben.

Con base en la revisión bibliográfica oportunamente citada, se encontró en el caso de Kenya, que los costos económicos que implica la informalidad empresarial son inferiores a los beneficios que ella genera, favoreciendo la mayor participación de su economía en el comercio internacional.

Una consideración concluyente es que a pesar de que la informalidad genera en Colombia competencia desleal a comerciantes y productores formales, así como evasión fiscal, estos son problemas menores frente al gran problema de la pobreza al cual contribuye a mitigar. Además se observa que la informalidad evita la profundización de los niveles de pobreza relativa en fases de lento crecimiento económico.

Se observa en la economía colombiana un comportamiento similar al de otras economías latinoamericanas, que indican que en el largo plazo el coeficiente de desigualdad en la distribución del ingreso (Gini) aumenta de manera muy correlacionada con el PIB.

A nivel macroeconómico a partir del análisis con un modelo teórico post-keynesiano, se obtienen conclusiones que corroboran algunas de las ya señaladas. Una de ellas es que el ingreso de los trabajadores informales, efectivamente contribuye al crecimiento del empleo formal y a la reducción del desempleo de equilibrio, a través del efecto multiplicador de su ingreso y su gasto. Este efecto se genera en parte por la alta importancia relativa del gasto en consumo de los trabajadores informales e independientes, que llegó a ser en promedio alrededor del $40 \%$ del consumo de los hogares colombianos en el periodo 1994/05, efecto similar en el caso de otros países como la india.

De otra parte las cadenas productivas en las que existe colaboración entre la industria formal 
e informal generan fuerzas que estimulan el crecimiento de los dos sectores.

Se verifica con cifras de 13 áreas metropolitanas de Colombia que el ingreso promedio por trabajador informal fluctúa alrededor del 50\% del ingreso promedio de los trabajadores formales, lo que representa una capacidad de gasto muy importante para mantener la estabilidad micro y macroeconómica.

\section{REFERENCIAS}

Bigsten, A., Kimuyu, P. \& Lundvall, K. (2004) What to do with the informal sector? En Development Policy Review, Volumen 22(6), Ps. 701-715. Disponible en: http://www. economics.handels.gu.se/digitalAssets/1378/1378664_pkkl-dpr-2004.pdf

Castañeda, A. \& García, J. (2007) Hábitat y espacio público: el caso de los vendedores informales en el espacio público físico de Bogotá, Bogotá, Colombia: Alcaldía Mayor de Bogotá, Instituto para la Economía Social, PNUD, ONU-Hábitat.

Cimoli, M., Primi, A. \& Pugno, M. (2006) Un modelo de bajo crecimiento: la informalidad como restricción estructural. Revista de la CEPAL No. 88 (abril), Ps. 89-107.

De Soto, H. ET AL. (1987) El otro sendero. La revolución informal. Bogotá, Editorial La Oveja Negra.

Galvis, L. (2012) Informalidad laboral en las áreas urbanas de Colombia, documento de trabajo, Cartagena, Publicaciones del Banco de la República.

Harris, J. (1990) Linkages between formal and informal sectors in developing countries: A Review. Geneva, ILO WEP 2-19/WP.50.

Huberman, L. (2013) Los bienes terrenales del hombre. Bogotá, Editorial Panamericana.

Editorial (19 de agosto de 1996) Ingresos de los trabajadores informales crecen menos. Bogotá, Periódico Portafolio.

Ishengoma, E. \& Kappel, R. (2006) Economic Growth and Poverty: Does Formalization of Informal Enterprises Matter? Working paper. Leibniz, German Institute for Global and Area Studies.

Janneke, P., Moreno, A., \& Abdul, A. (2012) Subcontracting and the Size and Composition of the Informal Sector: Evidence from Indian. Bonn, Institute for the Study of Labor (IZA).
Klein, E. (1984) Los sindicatos o el sector informal. Bogotá, Nueva Sociedad No. 70 (enero- febrero), Ps. 95-101.

Maloney, W. (2004) Informality revisited, World Development, Volumen 32 (7), Ps. 1159-1178.

Morley, S. (2002) The effects of growth and economic reform on income distribution in Latin America. Revista CEPAL, No. 71 (agosto), Ps. 3-40.

Novick, M. (2008) Recuperando políticas públicas para enfrentar la informalidad laboral. El caso argentino 20032007. Bs Aires. Power Point. Ministerio de Trabajo, Empleo y Seguridad Social.

Piore, M. \& Sabel, Ch. (1984) The second industrial divide: Possibilities for prosperity, N.Y., Basic Books.

Portes A. \& Haller, W. (2004) La economía informal. CEPAL, Santiago de Chile.

Ranis, G. \& Stewart, F. (1999) V-Goods and the Role of the Urban Informal Sector in 
LÍNEA DE INVESTIGACIÓN: ECONOMÍA SOCIAL

Development, Economic Development and Cultural Change, Volumen 47 (2), Ps. 259-88.

Sandoval, G. (1992) Teoría del dinero, el ahorro y el desempleo de equilibrio. Ediciones ARFO, Bogotá.

Semana.com (13 de marzo de 2010) ¿Por qué Colombia no sale del Club de los Pobres?. Revista SEMANA. Disponible en: http://www. semana.com/nacion/articulo/por-que-colombiano-sale-del-club-pobres/114313-3
Sierra, O. (2001) Mercado laboral urbano y desempleo en los estratos bajos en Colombia. Economía colombiana y coyuntura política, CGR, 184(junio), 31-35.

Tokman, V. (1978) Una exploración de la naturaleza de las interrelaciones entre los sectores informal y formal. Rev. De la CEPAL, No. 96 (primer semestre).

Wilaber. (2009) Desarrollo o informalidad, Portafolio, Bogotá, noviembre 11. 


\section{ANEXO 1. ANÁLISIS DE CORRELACIÓN}

Dependent Variable: GINI

Method: Least Squares

Date: 04/20/14 Time 16:18

Sample: 19832005

Include observations: 23

\begin{tabular}{|l|c|c|c|c|}
\hline \multicolumn{1}{|c|}{ Variable } & Coeficient & \multicolumn{1}{c|}{ Std Error } & t-Statistic & Prob. \\
\hline PIB & 0.002554 & 0.000265 & 9.644409 & 0.0000 \\
\hline C & 0.336267 & 0.017516 & 19.19790 & 0.0000 \\
\hline R-squared & 0.815813 & Mean dependent var & 0.501845 \\
\hline Adjusted R-squared & 0.807042 & S.D. Dependent var & 0.037908 \\
\hline S.E. of regression & 0.016652 & Akaike info criterion & -5.269645 \\
\hline Sum squared resid & 0.005823 & Schwarz criterion & -5.170906 \\
\hline Log likelihood & 62.60092 & Hannan-Quinn criter. & -5.244813 \\
\hline F-statistic & 93.01463 & Durbin-Watson stat & 0.755900 \\
\hline Prob(F-statistic) & 0.000000 & & \\
\hline
\end{tabular}

\section{ANEXO 2. ANÁLISIS DE CAUSALIDAD}

Pairwise Granger Causality Tests

Date: 04/28/14 Time: 22:18

Sample: 19832005

Lags: 2

\begin{tabular}{|l|c|c|c|}
\hline \multicolumn{1}{|c|}{ Null Hypothesis: } & Obs & F-Statistic & Prob. \\
\hline PIB does not Granger Cause GINI & 21 & 3.33335 & 0.0616 \\
\hline PIB does not Granger Cause PIB & & 1.93252 & 0.1771 \\
\hline
\end{tabular}

